\title{
Avances y retrocesos del cooperativismo mexicano durante el periodo neoliberal
}

\author{
Claudia Elena Robles Cardoso ${ }^{1}$ \\ Universidad Autónoma del Estado de México
}

Sumario: I. Introducción. II. Antecedentes del cooperativismo en México. III. Globalizacion, neoliberalismo y cooperativismo. IV. Avances y areas de oportunidad del cooperativismo. V. Conclusiones. VI. Bibliografía.

Resumen. La sociedad actual se encuentra en un proceso de reconfiguración consecuencia de la globalización y el neoliberalismo. En este espacio nos daremos a la tarea de identificar los avances y retrocesos que presenta el cooperativismo ante los cambios que sufre nuestro país ante la propuesta de una economía diferente. Se tiene como objetivo redelinear como el movimiento del cooperativismo no sólo posee la parte emancipadora en práctica y discurso, sino que establece una serie de detonantes que propician de una manera más rápida su aplicación y apropiación. Surge así la propuesta de una economía diferente, es decir, la Economía Social, está se desarrolla a partir de la idea del bienestar colectivo y de la supremacía del humano sobre el capital.

Palabras clave: cooperativismo. Globalización, neoliberalismo, economía social.

Abstract. Today's society is in a process of reconfiguration because of globalization and neoliberalism. In this space we will undertake the task of identifying the advances and setbacks that cooperatives present in the face of the changes that our country suffers from the proposal of a different economy. The objective is to redefine how the cooperatives movement not only has the emancipating part in practice and discourse, but also establishes a series of triggers that facilitate its application and appropriation in a more rapid way. Thus, arises the proposal of a different economy, that is, the Social Economy, it is developed from the idea of collective well-being and the supremacy of human over capital.

Keywords: cooperatives. Globalization, neoliberalism, social economy.

1 Doctora en Derecho por la Universidad Autónoma del Estado de México e Integrante del Cuerpo Académico Estudios en Derechos Humanos y sus garantías. 


\section{Introducción}

La desigualdad en México se observa por el resquebrajamiento paulatino de la estructura social y económica, esta crisis generalizada nos hace voltear los ojos hacia la creación de empresas cooperativas y autogestionarias como alternativas de solución.

Las cooperativas son un modelo que desafía las formas individualistas y capitalistas de producción y distribución de bienes, servicios y créditos; se constituyen como empresas de participación social o familiar que se caracterizan por su humanismo y solidaridad.

La figura del cooperativismo contiene en si misma valores y principios éticos que la hacen distinta de las demás empresas, toda vez que las finalidades que persigue son totalmente diferentes. Estos principios en el año de 1995 fueron reiterados por la Alianza Cooperativa internacional en un congreso celebrado por motivo de sus cincuenta años de existencia

El presente trabajo tiene como objetivo fundamental llevar a cabo un análisis de los avances y retrocesos que ha tenido el cooperativismo en México en el periodo neoliberal.

\section{Antecedentes del cooperativismo en México}

El movimiento cooperativista comienza en México en la década de 1870 (Rojas, 2008, 2014) a partir de la creación de una cooperativa de Producción y Venta de sombreros en 1872; básicamente le toca el nacimiento, apogeo y declive del porfiriato.

El cooperativismo fue una idea novedosa venida de Europa, que albergo los sueños de los obreros de la industria en México, dado que, sometidos a la esclavitud del capital, lograron formar rápidamente el Circulo Obreros de México, organizando posteriormente (septiembre de 1873) el Primer Taller Cooperativo del cual surgiría la Primera Sociedad Cooperativa de México, integrada por un reducido número de 26 sastres de la Ciudad de México.

A pesar de la «paz» que existía en México, las cooperativas tuvieron muchos obstáculos constantes en todo el país: la indiferencia, la falta de organización, los intermediarios en el ámbito de la producción, la constante lucha por un nicho dentro del mercado y los fines (en algunos casos) políticos de los cuales eran objetos. Hasta antes de iniciada la Revolución Mexicana, el movimiento comenzaba a tener un peso y fuerza, pero éstos se vinieron abajo debido al movimiento de rebelión social. Este movimiento casi hizo que desaparecieran las cooperativas, no obstante, pasaron a ser especie de apéndice del movimiento obrero 
mexicano que tomó mucha fuerza en esos años de lucha. Afirma Rojas (1982, p. 376) «era tal la fuerza moral del cooperativismo que los líderes obreros y el gobierno mismo no pudieron sustraerse a su influencia».

En el periodo de la construcción del Estado mexicano (1920-1934), las cooperativas comenzaron a mostrar un crecimiento relativo mínimo, esto debido a que las consecuencias de la Revolución Mexicana dieron por hecho la necesidad de reconstruir el país. En este sentido, se puede observar el crecimiento de las cooperativas durante el periodo 1927- 1932.

Estas ideas de cooperación y ayuda mutua tuvieron en nuestro país un efecto muy particular pues al ser defendidas por los socialistas utópicos franceses, así como por ideólogos anarquistas, vinieron a constituir lo que en nuestra tradición existía en las comunidades indígenas como el trabajo colectivo y la propiedad comunal de las tierras en los famosos: «calpullis».

En consecuencia, puede afirmarse que las instituciones de tipo cooperativo que surgieron en México nacieron en medio de grandes luchas sociales y agitaciones populares en defensa de tales ideas, identificándose desde sus inicios con los movimientos: campesino y obrero (1800 a 1903).

En México, el cooperativismo no es un tema nuevo, de hecho, se formalizó desde 1927 con la expedición de la Primera Ley General de Sociedades Cooperativas, aunque los avances en la materia se dieron en la última década con la aprobación de la Ley para Regular las Actividades de las Sociedades Cooperativas de Ahorro y Préstamo en 2009.

Por lo que el cooperativismo mexicano, a lo largo de su historia no pasó de ser un movimiento de dimensiones minúsculas, opacado por el sindicalismo, y cuyos telones de fondo, la intervención estatal y la desorganización de la clase obrera, incidieron más en su lento desarrollo que en el éxito de este.

Lo cual conduce a observar una característica fundamental del cooperativismo mexicano, éste más que ser propiciado en el seno de las masas, es las más de las veces, una política, una ideología o un experimento impulsado desde arriba.

Sin embargo, pese a haber sido una forma de organización con dimensiones más pequeñas que las del sindicalismo, el cooperativismo es fundamental para entender el desarrollo de la clase obrera en México, ya que involucra no sólo la ideología, sino las formas de negociación entre clases. Es interesante observar cómo este sistema de organización se convierte en un instrumento de legitimación de poder y control estatal. Por tanto, su contribución, más allá de ser cuantitativa (unidades de producción, socios en cooperativas, producción, etc.), es cualitativa (su legado ideológico y la preservación de éste hasta la actualidad. 


\section{Globalizacion, neoliberalismo y cooperativismo}

Podemos decir que la globalización es «ampliación, profundización y aceleración de la interconexión mundial en todos sus aspectos de la vida social contemporánea» (Lerman, 2006, p. 9), sin embargo, esta definición carece de profundidad y delimitación en sí misma, por lo que le agregaremos que la ampliación es con referencia al capital financiero (llevado a cabo por la especulación en las bolsas internacionales), la profundización que se da en el seno de las desigualdades sociales tanto dentro de los Estado-Nación como en sus interacciones y la aceleración, a través de las empresas trasnacionales, para el cumplimiento del único objetivo del sistema: la acumulación de capital. Así la globalización busca, mediante la persuasión discursiva, posicionar al neoliberalismo como el eje principal del desarrollo.

Etxezarreta (2001, p. 13) menciona que la globalización «no es más que el nombre que se da a la etapa actual del capitalismo». Por otro lado, Fernand Braudel (1986) afirma que el capitalismo comenzó a incubarse desde el siglo doce como resultado del mercantilismo que se practicó en ese momento; de allí se dio paso a la Revolución Industrial que dio como resultado que se comenzara a expandir no sólo la producción de mercancías (bienes y servicios), sino también la práctica de la relación tripartita entre el capital, el capitalista y el obrero.

Mientras se explicaba y describía el proceso de acumulación de capital y el proceso de generación de riqueza, surgieron dos corrientes que entendían y explicaban estas nuevas relaciones sociales. La primera explicaba que la generación de riqueza se hacía a través de la reconfiguración que se da por parte de las materias primas y por ende de las máquinas, mientras que la segunda explicaba que la generación de riqueza se encontraba en la mano de obra que se empleaba para la construcción de los productos.

Las leyes económicas, referidas al mercado implican una serie de restricciones hacia el Estado, es decir, a limitar su posible intervención en el ámbito económico. De allí que a partir de los tratados y modificaciones legales que se dieron por toda América Latina, el Estado permitiera el establecimiento de capital extranjero (empresas trasnacionales) que «ayudaría a incrementar la producción» para exportar los bienes y generar riqueza.

Todo el proceso capitalista se ha enfocado, hasta este momento, en la maximización de la producción y en la importancia de liberar el capital de cualquier traba, por lo que a este punto del capitalismo se ha llamado globalización.

Las relaciones sociales del trabajo actualmente están regidas por el paradigma neoliberal, que cobró fuerza en la década de 1980 cuando 
el Estado de bienestar se enfrentó a su mayor crisis debido a su incapacidad de velar por la seguridad de los trabajadores y por los intereses propios del sector privado.

La Crisis Económica y Financiera que golpea se agrava cada día más, resultado de la conjugación de los procesos de globalización con la práctica de los modelos neoliberales, acentuando la injusta distribución de la riqueza.

La Globalización Neoliberal ha tratado de convertir los servicios sociales en transacciones de mercado, los ciudadanos en consumidores y las necesidades inalienables en demanda efectiva. Es por esta razón que la década de los 90 devino una década muy convulsa, pero a su vez con imperiosa necesidad de plantear alternativas de solución a la crisis global que nos afecta.

En este sentido hay que destacar que en este contexto y sobre todo a partir de los años 80 se presenta para los países latinoamericanos un nuevo sector de economía, una alternativa de desarrollo económica, productiva y social. La Economía Solidaria, Social o del Trabajo que surge en medio de una fuerte ofensiva neoliberal. Siendo sus componentes más importantes: "Las Cooperativas», Mutuales, Fondos de Empleados, etc. Corresponde entonces al cooperativismo a partir de nuestro criterio el lugar esencial siendo sin lugar a duda, el movimiento socioeconómico más grande del mundo y que sobre todo humaniza al hombre

El cooperativismo hoy se expande no solo en los países tercer mundistas, sinoque también se manifiesta en países desarrollados, estando presente en todas y cada una de las ramas de la economía, por supuesto acorde a las peculiaridades de cada país.

Se consolida entonces como un reto para el sector solidario generar estrategias que les permitan posicionarse competitivamente en el mercado al tiempo que fortalecen y promueven su identidad particular, especialmente desde la consolidación de los principios solidarios; el reto es en relación a que debe generar diálogos entre la economía solidaria y el contexto neoliberal, logrando un equilibrio que fortalezca la dimensión social de las organizaciones, al tiempo que se favorece una organización del trabajo que promueve la calidad de vida, pero que se torna competitiva en el mercado.

\section{Avances y areas de oportunidad del cooperativismo}

Se debe entender que cooperativismo proviene del verbo cooperar y éste del latín cooperare, el cual según el diccionario significa «Obrar conjuntamente dos o más personas o entidades para conseguir un 
mismo fin» (Larousse, 2010, p. 287). Esta definición es la que da como carácter principal al movimiento; la idea de la acumulación de riqueza no se encuentra dentro del fin último del cooperativismo, al contrario, y como se verá más adelante, es dar una mejor calidad de vida.

Las cooperativas en México son un motor económico indispensable para la nación: generan empleo, aportan al desarrollo de las comunidades y facilitan el acceso de la población a distintos servicios y productos financieros que les mejoran lavida.

Algo que particularmente llamó mi atención fue el hecho de que la cooperación, como un medio de asociación capaz de elevar el nivel de vida de las clases más bajas, fuera adquiriendo relevancia económica conforme avanzaba el capitalismo

Las condiciones del entorno económico financiero, como consecuencia fundamental de la globalización de la economía, tienen un impacto directo sobre las estrategias de las organizaciones, y por lo tanto, en las cooperativas. Por otro lado, ante los dilemas que se generan como consecuencia de vivir en contextos globalizados, la pobreza es ahora una realidad global que requiere de soluciones locales; en este sentido Arocena (1995) ha señalado, que lo local se ha concebido como «el remedio universal de todos los males».

Las organizaciones cooperativistas juegan un papel relevante en el desarrollo local, ya que a través de ellas es posible llevar a cabo actividades para el beneficio de las personas que integran una comunidad, organizándose en torno a la gestión empresarial que no tiene como razón principal la maximización de ganancias, sino la satisfacción de ciertos objetivos económicos y sociales. Su creación y puesta en marcha favorece la inserción social y laboral entre los sectores más desprotegidos, atenuando la marginación y pobreza mediante la producción de bienes o servicios (Lara \& Rico, 2011).

En México se encontraron como principales representantes de este sistema a las cooperativas de ahorro y crédito, de producción agrícola y de producción pesquera. Algunos ejemplos de localidades que se han beneficiado con este modelo cooperativista son: Balancán, Tabasco Pijijiapan, Chiapas Oxchuc, Chiapas Pinal de Amoles, Querétaro Hermosillo, Sonora Monterrey, Nuevo León. Ampliando lo anterior, uno de los rubros más representativos dentro de las cooperativas en México son las de ahorro y crédito, las cuales se encuentran en el centro del país, ubicadas en primer lugar en el estado de Guanajuato con 692,578 socios, en segundo, Querétaro con 336,577 socios y en tercer lugar en el estado de Jalisco con 272,565 socios (Lara, et al. 2011). Para el año 2012 y de acuerdo con la Comisión Nacional Bancaria y de Valores (CNBV), se encontraban en operación 63 cooperati- 
vas de ahorro y crédito en México, así como 6 más autorizadas, pero sin operación.

El movimiento cooperativista en México ha tenido sus altas y bajas. En realidad, las condiciones sociales de México, que van desde su Independencia hasta el momento actual, generan una especie de ambiente hostil en el que el movimiento cooperativista ha desarrollado su base de acción. México se ha mantenido en una guerra interna desde la consumación de su Independencia; esto se ha debido a las disputas por detentar el poder y la administración de un país lleno de riqueza.

La guerra vivida en México se ha caracterizado por una relación de poder basado en el nepotismo (dentro de la clase política), en el clientelismo, corporativismo y sindicalismo (desde la clase política a la trabajadora) y de individualismo y competencia (dentro de la población en general). La relación histórica entre la clase política y la población trabajadora mexicana se ha enfocado en una relación dependiente que genera, por un lado, la reproducción del statu quo y por el otro, los privilegios de ciertos individuos de la población en general (los dirigentes de sindicatos, organizaciones campesinas y obreras, por ejemplo).

Esto, aunado a las condiciones microeconómicas de la población (salarios bajos que atraen la inversión extranjera), así como la no erradicación de los grandes problemas nacionales como lo es la educación, la pobreza, la desigualdad social, el racismo y la violencia generada por el Estado y el crimen organizado, hacen que muchas veces las cooperativas sólo sean de forma y no de hecho.

En México diversos factores restringen el desarrollo económico, siendo la pobreza de ingresos uno de ellos; pero además existen otros como el limitado acceso a los mercados financieros, la precaria salud de los habitantes, la educación de baja calidad y la inestabilidad en el campo laboral. Para revertir los efectos nocivos de la marginación, el gobierno y otros actores sociales han instrumentado estrategias relacionadas con la creación de empresas sociales, que en su mayoría se han legitimado a través de cooperativas, cuyos integrantes basan sus prácticas en la filosofía cooperativa. Su creación y puesta en marcha favorece la inserción social y laboral entre los sectores más desprotegidos, atenuando la marginación y la pobreza mediante la producción de bienes o servicios (Lara \& Rico, 2011).

De esta forma, una de las principales actividades de este sistema son las cooperativas de ahorro y préstamo en México, las cuales surgen como una de las opciones para generar oportunidades y dinamizar la economía con una visión centralizada en la persona y el desarrollo de las comunidades, ya que para atender problemáticas tan complejas 
y revertir los efectos de la marginación hace falta la colaboración decidida de los actores públicos, privados y sociales.

Se reconoce que una buena parte de los ingresos de las comunidades de México provienen de las remesas de los que trabajan en el extranjero, las cooperativas son reconocidas por los beneficios que prestan a la población a través del financiamiento accesible; por otro lado, los directivos consideran que mediante el crédito se impulsa la creación y desarrollo de empresas locales, opinan que el principal aporte de las cooperativas es que se estimula el carácter emprendedor de los socios, pues se han creado microempresas de diversas actividades con las que se ha fomentado el autoempleo y la creación de nuevas fuentes de trabajo, lo que lleva a mejorar económicamente a los habitantes (Lara \& Rico, 2011).

Las cooperativas de ahorro y crédito constituyen una estrategia de desarrollo local que produce un avance social y económico de las comunidades marginadas, en tanto formen parte de un proyecto integral que incluya estrategias para la mejora social y económica de sus habitantes (Lara \& Rico, 2011). Un ejemplo exitoso de esta visión de empresa social lo indica Verónica de la O citada en Cano et al. (2012), en su estudio con cooperativas pesqueras en Baja California, México; en términos de la contribución de éstas hacia el crecimiento económico de esa entidad y del país. Hoy en día, al convertirse en sociedades cooperativas pesqueras sustentables que han logrado la autonomía en cuanto a la comercialización de sus productos a través de un largo proceso de aprendizaje sobre el mercado internacional, sorteando el abandono por parte del Estado, al quitarles la concesión de especies de alto valor comercial, y el conocimiento sobre el recurso que los mantiene a flote. Las cooperativas pesqueras se consideran empresas sociales que en economías de mercado cumplen funciones redistributivas cuando se rodean de un ambiente propicio que les permite operar con la singularidad que las caracteriza; comprenden un amplio rango de entidades productivas y de servicios donde el beneficio de la actividad económica que realizan se reparte entre varios individuos, generalmente los mismos que trabajan en ellas. El estudio de las cooperativas bajo el enfoque de empresa social, por lo tanto, implica un acercamiento desde dos grandes aspectos:

1. La conformación del entorno y 2) las características propias de dicha empresa social (Cano, et al., 2012).

2. Algunos de los puntos que detienen el desarrollo de las cooperativas en México son la falta de organización y la resistencia al cambio; donde se puede resaltar la importancia del desarrollo organizacional ya que permite tener más acceso a la obtención 
de recursos económicos, mejorar la infraestructura, volver eficiente el uso del recurso, que se traduce en la conservación del mismo y la aceptación de nuevas formas de innovación tecnológica, ejemplo de ello es el caso de la Federación de Cooperativas Pesqueras de Baja California, cuyo producto es la langosta roja, está certificado por el Consejo para la Administración Marina (MSC por sus siglas en ingles), por contar con una buena capacidad organizativa (equilibrio en la organización y el grado de tecnificación) que le permitió convertir su producto en una pesquería sustentable y bien administrada (Cano, et al., 2012). Otro problema frecuente de las cooperativas es que en muchas ocasiones los grupos de poder poseen los medios económicos y el dominio social y por lo mismo son elegidos erróneamente para ser líderes de proyectos. Como resultado, estos proyectos no tienen un impacto en el combate a la pobreza, más bien intensifican las diferencias económicas ya existentes, incrementando las riquezas de los menos necesitados y la vulnerabilidad de la mayoría (Sabatés, 2012). Si los individuos que participan en un proyecto de desarrollo lo hacen de manera voluntaria, esto significa que tienen la motivación necesaria para hacerlo, entonces existe la «voluntad participativa»; pero si al contrario las personas se sienten obligadas a participar en los proyectos de desarrollo, entonces el resultado es una participación inactiva o apática. Uno de los pilares que brinda sustentabilidad a los proyectos es la manera en la cual los administradores o dirigentes y los miembros de los proyectos reaccionan ante estos acontecimientos y se logran ajustar ante las situaciones imprevistas (Sabatés, 2012).

Asimismo, de acuerdo con Herrera (2012) sí los más de 80,000 millones de pesos en activos que tienen en su capital social las cooperativas mexicanas de ahorro y crédito, se orientaran a la inversión productiva, se lograría un importante fortalecimiento y ampliación del mercado interno con beneficios inmediatos para la población de escasos recursos, con esto las sociedades cooperativas de ahorro y préstamo estarían realizando una importante aportación al desarrollo nacional que hoy, ni el gobierno ni los empresarios privados, son capaces de realizar. En definitiva, la mejor estrategia para promover el fortalecimiento del movimiento cooperativo mexicano no es promoviendo su alineamiento en condiciones de subordinación y mero complemento del sistema financiero mexicano, sino preservando su identidad asociativa y su cultura empresarial de naturaleza solidaria y democrática. Para 
lograr esta tarea de orden estratégico lo que se demanda de los legisladores es disposición para escuchar, apertura para aprender y sensibilidad para legislar y de los cooperativistas firmeza en la defensa de su identidad cooperativa, capacidad para comunicar sus logros empresariales y sociales y disposición para consensuar con la clase política (Herrera, 2012).

Uno de los problemas más importantes con el que el movimiento cooperativista se ha encontrado en México es sin duda la falta de organización entre la sociedad mexicana; según datos del Centro de Investigaciones Económicas Sociales y Tecnológicas de la Agroindustria y la Agricultura Mundial (CIESTAAM) «El 85\% de los mexicanos no ha participado en grupos organizados, lo cual es un fiel reflejo de la debilidad de nuestro tejido social» (Muñoz, Santoyo \& Flores, 2010, p. 14). Dejando por un momento este obstáculo organizativo, nos encontramos con el problema de la teoría en México; desde el comienzo de la aplicación de las cooperativas en México, hemos tenido una influencia externa (de Alemania, por ejemplo) que lejos de adaptarse a las condiciones mexicanas se hace al revés: la sociedad debe modificarse ante los requisitos que solicita el modelo extranjero. Es por ello que la escasa producción bibliográfica que hay en México y finalmente el problema que representa el sistema económico imperante tanto en el país como en el resto del mundo (excluyendo a China y Cuba) ya que con los principios que se rige la globalización es muy difícil que la gente adquiera y asimile los principios cooperativistas.

Dentro de las cooperativas más representativas en México se encuentran la «Unión de Cooperativas Tosepan» (1977) y la «Sociedad Cooperativa Trabajadores de Pascual» (1985). Ambas creadas como resultado de condiciones políticas y económicas adversas a la población (indígenas y obreros); en este sentido, es importante mencionar que estas dos cooperativas se han conformado como ejemplos clásicos de cómo una cooperativa puede mantenerse en el tiempo.

No hay que olvidar que una cooperativa no se conforma como un proyecto a corto plazo, sino como un proyecto de vida que tiende a una prospectiva de corte generacional. Esto es algo que muchas cooperativas no han cuidado y que, por ende, trae problemas en la segunda generación debido a que ellos no han sido formados con los valores cooperativistas; no han sido educados para ser cooperativistas. Este problema lo está viviendo la cooperativa Pascual, debido a la falta de apropiación histórica por parte de las nuevas generaciones sobre cómo se conformó dicha sociedad cooperativa, lo que ha desembocado en que haya constantes intentos de venderla para adquirir una ganancia mayor sin tomar en cuenta el esfuerzo y la lucha concentrada en esta organización. 
La cooperativa Tosepan ha sabido llevar este tipo de problemas con la educación cooperativista que da a los hijos de los socios e incluso a través de su inserción desde chicos para que la valores y se sientan parte de ella. Además, esta cooperativa reivindica los valores, usos y costumbre del grupo indígena que lo conforma, lo que no sólo es la conservación de una organización y fuente de empleo, sino de una cultura.

Las cooperativas administradas y manejadas por grupos indígenas tienen un factor que ayuda a mantener el proyecto a largo plazo: su cohesión sociocultural. Es decir, a partir de la cultura y el proceso histórico que los ha llevado a atenerse en una posición de hermetismo cultural, han logrado mantener un espacio idóneo para la proliferación de proyectos donde se hay confianza y compromiso. Muchos de los valores cooperativos son similares a los generados en las comunidades indígenas, donde la solidaridad y el apoyo común sin recibir nada a cambio, generan relaciones sociales de confianza y cooperativismo.

En el 2012 es cuando se crea la Ley General de Economía Social y Solidaria. Aunque existe polémica sobre lo benéfica de esta nueva Ley sobre la Economía Social y Solidaria, no cabe duda de que este interés cada vez más pronunciado por parte de la sociedad mexicana por este tipo de legislaciones que reivindican el movimiento y le darán más fuerza para consolidarse.

El artículo primero de la presente Ley señala que es reglamentaria del párrafo séptimo del artículo 25 de la Constitución Política de los Estados Unidos Mexicanos en lo concerniente al Sector Social de la Economía, es de orden público, interés social y de observancia general en todo el territorio nacional y se aplicará sin perjuicio de otras disposiciones que dicten los Poderes Ejecutivo y Legislativo Federal y de las Entidades Federativas, así como municipales, en el ámbito de sus respectivas competencias.

El objeto de la presente Ley señala el artículo segundo es:

I. Establecer mecanismos para facilitar la organización y la expansión de la Actividad Económica del Sector Social de la Economía y la responsabilidad del fomento e impulso por parte del Estado.

II. Definir las reglas de organización, promoción, fomento y fortalecimiento del Sector Social de la Economía, como un sistema eficaz que contribuya al desarrollo social y económico del país, a la generación de fuentes de trabajo digno, al fortalecimiento de la democracia, a la equitativa distribución del ingreso y a la mayor generación de patrimonio social. 
El Sector Social de la Economía es el sector de la economía a que hace mención el párrafo séptimo del Artículo 25 de la Constitución Política de los Estados Unidos Mexicanos, el cual se integra por el conjunto de organizaciones sociales en los términos que establece la presente Ley.

El artículo cuarto nos señala: «el Sector Social de la Economía estará integrado por las siguientes formas de organización social:

I. Ejidos;

II. Comunidades;

III. Organizaciones de trabajadores;

IV. Sociedades Cooperativas;

V. Empresas que pertenezcan mayoritaria o exclusivamente a los trabajadores; y

VI. En general, de todas las formas de organización social para la producción, distribución y consumo de bienes y servicios socialmente necesarios.

La Economía Solidaria, Social o del Trabajo que surge en medio de una fuerte ofensiva neoliberal, su esencia se sustenta en cinco principios fundamentales:

1. La solidaridad, la cooperación y la democracia como forma de vida y de convivencia humana, norma que debe cumplir toda persona y organización laboral y empresarial que hace parte del sector de la Economía Solidaria.

2. La supremacía del trabajo sobre el capital,

3. El trabajo asociado como base fundamental de la organización de la empresa, la producción y la economía,

4. La propiedad social de los medios de producción

5. La Autogestión como forma superior de la participación de los trabajadores en la gestión de la empresa, la economía y en la conducción de la sociedad y el Estado, siendo sus componentes más importantes: «Las Cooperativas», Mutuales, Fondos de Empleados, etc.

Presenta una importante experiencia teórica y práctica de desarrollo del llamado sector solidario de la economía en general y del cooperativismo en particular, donde se revelan un grupo de importantes particularidades, que a su vez devienen retos a enfrentar en el nuevo milenio, estas son:

1. Pérdida de la naturaleza y esencia cooperativa.

2. Cooperativismo financiero de Ahorro y Crédito cuantitativamente fuerte y cualitativamente débil. 
3. Cooperativismo Agropecuario muy débil.

4. Cooperativismo en los Servicios muy diversificado

5. Deficiente estructura del cooperativismo.

6. Marco legal inadecuado y deterioro en las relaciones EstadoCooperativas.

7. Deficiente proceso de Educación.

8. Falta de Integración.

Como todo movimiento, el cooperativismo no sólo posee la parte emancipadora en práctica y discurso, sino que establece una serie de detonantes que propician de una manera más rápida su aplicación y apropiación. Surge así la propuesta de una economía diferente, es decir, la Economía Social. Ésta se desarrolla a partir de la idea del bienestar colectivo y de la supremacía del humano sobre el capital

\section{Conclusiones}

- Los efectos de la actual crisis económica y financiera han acentuado la ya profunda brecha entre riqueza y pobreza condicionando la necesidad de buscar alternativas de salida a esta situación.

- El Cooperativismo en México enfrenta hoy importantes retos ante la actual Globalización Neoliberal los que debe asumir y resolver, en su aspiración de convertirse en una real alternativa de desarrollo económico, productivo y social.

- Las cooperativas tienen un papel trascendental para las actividades económicas donde el negocio central es intrínseco en la comunidad y parte de la identidad de ésta; es decir, donde las principales actividades de las cooperativas son parte del conocimiento y dominio popular de la comunidad; asimismo, que los beneficios de una cooperativa fortalecen la actividad económica de la zona y son distribuidos equitativamente.

- Por ello, es importante resaltar la urgencia por consolidar investigaciones sobre el cooperativismo para poder hablar sobre nuestras experiencias y crear espacios de diálogo. El diálogo es una de las opciones más viables para hacer ver a los individuos que otro tipo de sociedad es diferente. Dice el dicho popular que «nadie escarmienta en cabeza ajena», sin embargo, lo cierto es que se puede evitar, teniendo el mecanismo idóneo para ello. 


\section{Vl. Bibliografía}

ArocenA, José (1995) El desarrollo local, un desafío contemporáneo. Uruguay: Nueva Sociedad, CLAEH y Universidad Católica del Uruguay, Caracas.

Bauman, Z. (2010). La globalización. Consecuencias humanas. México: Fondo de Cultura Económica.

Chossudovsky, M. (2002). Globalización de la pobreza y nuevo orden mundial. México: UNAM-Siglo XXI.

ETXEZARRETA, M. (2001). Algunos rasgos de la globalización. En R. Fernández Durán,

M. ETXEZARRETA, R. L.P, \& R. L. L. P., Globalización capitalista (p. 13-42). Barcelona: LUNA.

Fritz-Krockow, B. (1986). Evaluación del cooperativismo mexicano. Comercio Exterior, p. 789-796.

IANNI, O. (2006). Teorías de la globalización. México: Siglo XXI.

J. Hinkelammert, F., \& Mora Jiménez, H. (2013). Hacia una economía para la vida. Morelia: EUNA-Universidad Michoacana.

Larousse. (2010). El PEQUeÑo LaRousSe ILUSTRAdo 2010. México: LaRousse.

Lerman Alperstein, A. (2006). Relaciones México-América Latina. Entre la globalización y la regionalización. México: AUM-X.

Moura, V. (1946). Diretrizes cooperativistas. Sao Paulo: Editora Brasiliense Limitada.

Muñoz Rodríguez, M., Santoyo Cortés, V. H., \& Flores Verduzco, J. J. (2010). Pilares de las organizaciones rurales que perduran. México: UACH.

Revista Mexicana del Trabajo. Datos históricos sobre el cooperativismo en México. Quinta época. Tomo IX Méx. DF. 1972.

Rojas Coria, R. (1982). Tratado de Cooperativismo Mexicano. México: Fondo de Cultura Económica.

Rojas HerRera, J. J. (2008). Rosendo Rojas Coria: una vida dedicada al engrandecimiento del cooperativismo mexicano. México: UACh.

Rojas HerReRA, J. J. (2014). La formación del movimiento cooperativo en México: antecedentes organizacionales y momento constitutivo. México: UACh-Juan Pablos Editor.

Santos De Morals, C. (2004). Cuadernos cooperativos y de Economía Social. Apuntes sobre Teoría de la Organización. México: Cámara de Diputados.

SolidARIA, G. d. (2006). Cuadernos Cooperativos y de Economía Social. México: Cámara de Diputados.

Velázquez García, J. M. (2013). El movimiento cooperativo en México: La búsqueda de alternativa al desarrollo social 2000-2010. México: Movimiento Ciudadano.

Ley de la economía social y solidaria, reglamentaria del párrafo séptimo del artículo 25 de la Constitución Política de los Estados Unidos Mexicanos, en lo referente al sector social de la economía consultada en: http://www.diputados.gob.mx/LeyesBiblio/ref/less/LESS_orig_23may12.pdf 


\section{Derechos de autor}

La revista Deusto Estudios Cooperativos es una revista de acceso abierto lo que significa que es de libre acceso en su integridad inmediatamente después de la publicación de cada número. Se permite su lectura, la búsqueda, descarga, distribución y reutilización legal en cualquier tipo de soporte sólo para fines no comerciales y según lo previsto por la ley; sin la previa autorización de la Editorial (Universidad de Deusto) o el autor, siempre que la obra original sea debidamente citada (número, año, páginas y DOI si procede) y cualquier cambio en el original esté claramente indicado.

\section{Copyright}

The Deusto Journal of Cooperative Studies is an Open Access journal which means that it is free for full and immediate access, reading, search, download, distribution, and lawful reuse in any medium only for non-commercial purposes, without prior permission from the Publisher or the author; provided the original work is properly cited and any changes to the original are clearly indicated. 